\title{
Microsoft Infosec Team: Security Tools Roadmap
}

\author{
Simon Roses \\ Microsoft, United Kingdom \\ simonros@microsoft.com
}

The Microsoft IT's Information Security (InfoSec) group is responsible for information security risk management at Microsoft. We concentrate on the data protection of Microsoft assets, business and enterprise. Our mission is to enable secure and reliable business for Microsoft and its customers. We are an experienced group of IT professionals including architects, developers, program managers and managers.

This talk will present different technologies developed by Infosec to protect Microsoft and released for free, such as CAT.NET, SPIDER, SDR, TAM and SRE and how they fit into SDL (Security Development Lifecycle). 